\title{
Creatinine clearance and subclinical target organ damage in primary hypertension
}

\author{
Das S K, Jha S C \\ Department of Internal Medicine, Institute of Medicine, Tribhuvan University Teaching Hospital \\ Correspondence to: Dr Santa Kumar Das, Institute of Medicine, Kathmandu, Nepal.
}

Email: drsantadas@hotmail.com

\begin{abstract}
Introduction: Despite the widely recognized dangers of uncontrolled hypertension, the disease remains inadequately treated in the majority of patients. This may be, in large part, because of the asymptomatic nature of the disease for the first 15 to 20 years, even as it progressively damages the cardiovascular system. Therefore, assessment of hypertension related subclinical target-organ damage represents a key diagnostic procedure for the risk stratification of hypertensive patients.

Methods: A prospective case control study of 40 cases (hypertensive patients with $\mathrm{CCR}<60$ ) and 40 controls (hypertensive patients with CCR $>60$ ) was conducted in Tribhuvan University Teaching Hospital (TUTH). Renal function was estimated by the Cockcroft-Gault formula. Left ventricular hypertrophy was determined by echocardiography. Retinal vascular changes were evaluated by direct ophthalmoscopy. Microalbumin in urine was measured from spot morning sample.

Results: The prevalence of Left ventricular hypertrophy (LVH), microalbuminuria and retinopathy in cases and control group was 55\% VS 20\% (P=.001), 50\% VS 20\% (P=.004) and $92.5 \%$ VS 52.5\% $(\mathrm{P}=.001)$. Patients with microalbuminuria showed prevalence of $\mathrm{LVH}, \mathrm{CCR}<60$ and retinopathy as $78.57 \%, 71.43 \%$ and $100 \%$ respectively. There was high prevalence of grade I and grade II retinopathy in patients with low CCR

Conclusions: Results show that a reduction in creatinine clearance and/or presence of microalbuminuria is a marker of subclinical organ damage in patients with primary hypertension and normal serum creatinine irrespective of BP load and other traditional risk factors.
\end{abstract}

Keywords: Creatinine clearance, primary hypertension, subclinical, target organ damage.

\section{Introduction}

As the population grows older and more obese, the incidence of hypertension continues to increase, not only in developed societies but also in developing societies. ${ }^{1} \mathrm{At}$ the same time, despite the widely recognized dangers of uncontrolled hypertension, the disease remains inadequately treated in the majority of patients. ${ }^{2}$

Asymptomatic patients are often unwilling to alter life style or take medication to forestall some far-off, poorly perceived danger, particularly when they are made uncomfortable in the process. Therefore, assessment of hypertension related subclinical target-organ damage represents a key diagnostic procedure for the stratification of hypertensive patients according to their cardiovascular risk profile. Recent Guidelines jointly issued by the European Society of Hypertension and the European Society of Cardiology (ESH-ESC) have emphasized the rationale, indications and therapeutic implications of this evaluation. ${ }^{3}$

Optimizing the diagnostic approach to the detection of target organ damage is of the utmost importance for a rationale and cost-effective allocation of economic resources. 
Although extensive diagnostic workup can lead to the identification of a larger number of patients at risk, routine Ultra sonogram evaluation of target organ damage may be too expensive and impractical to be applied on a large scale. The development of new, integrated, and low-cost tools to identify patients at higher risk may therefore prove to be very helpful in clinical practice.

This study is expected to help in improving the level of compliance in otherwise asymptomatic hypertensive patients by creating awareness regarding subclinical organ damage. This study is also expected to promote determination of Creatinine clearance rate (CCR) and microalbuminuria as routine procedure in evaluation of hypertensive patients, as these tests are easily available and less costly.

\section{Methods}

This is a prospective case control study conducted in department of internal medicine, Tribhuvan University Teaching Hospital, which is a tertiary care hospital in Nepal. We included diagnosed case of primary hypertension with normal serum creatinine. Patients with $\mathrm{CCR}<60$ were taken as cases and patients with CCR $>60$ as controls. All the included patients were divided into 2 groups, each containing 40 patients.

Hypertensive patients with overt organ damage, diabetes mellitus (DM), and severe obesity were excluded from study. Renal function was estimated by means of the serum creatinine level using the Cockcroft-Gault formula. This value was adjusted for body surface area (BSA). Left ventricular hypertrophy was determined by electrocardiography (Romhilt-Estes criteria) and echocardiography (Troy formula). Retinal vascular changes were evaluated by direct ophthalmoscopy. Microalbumin in urine was measured from spot morning sample after excluding other possible causes of microalbuminuria. All analysis was performed using statistical software (SPSS v 11.5).

\section{Results}

Among the study patients male to female ratio was $0.77: 1$. The mean age in control group was $53.70 \pm 9.72$ years, while in case group it was $64.03 \pm 8.50$ years. The mean duration of hypertension in control patients was $4.78 \pm 2.03$ and that in case was 7.08 \pm 3.54 . The mean CCR in control patients was $76.96 \pm 11.50 \mathrm{ml} / \mathrm{min}$ per $1.73 \mathrm{~m}^{2}$ and in case group it was $53.49 \pm 5.30 \mathrm{ml} / \mathrm{min}$ per $1.73 \mathrm{~m}^{2}$. The prevalence of left ventricular hypertrophy, microalbuminuria and retinopathy was significantly higher in patients with $\mathrm{CCR}<60$ (Table 1).
Table 1: Prevalence of Target organ damage (TOD) in study patients according to creatinine clearance rate

\begin{tabular}{lllllllll}
$\begin{array}{l}\text { Patients } \\
\text { withCCR }\end{array}$ & \multicolumn{2}{c}{ LVH } & \multicolumn{2}{c}{ LVDD } & \multicolumn{1}{c}{ MICROAL RETINO } \\
& Freq. & $\%$ & Freq. & $\%$ & Freq. & $\%$ & Freq. \% \\
$\geq 60 \mathrm{n}=40$ & 8 & 20 & 8 & 20 & 8 & 20 & 21 & 53 \\
$\leq 60 \mathrm{n}=40$ & 22 & 55 & 11 & 28 & 20 & 50 & 37 & 93 \\
r value & -0.361 & & & -0.314 & & & \\
pvalue & 0.001 & & NS & 0.004 & & 0.001
\end{tabular}

Similarly, left ventricular hypertrophy, decreased in renal function and retinopathy all were significantly higher in patients with microalbuminuria(Table 2).

Table 2: Prevalence of TOD in patients with microalbuminuria

$\begin{array}{lllllll}\text { Microal } & \text { LVH } & \text { CCR }<60 & \text { Retino } & & & \\ & \text { Freq. } & \% & \text { Freq. } & \% & \text { Freq. } & \% \\ (+)(n=28) & 22 & 79 & 20 & 71 & 28 & 100 \\ (-)(n=52) & 8 & 15 & 20 & 38 & 30 & 58 \\ \text { r value } & 0.677 & -0.314 & & & \\ \text { p value } & 0.000 & 0.004 & & 0.001 & \end{array}$

Among 80 study patients, 55 patients $(68.75 \%)$ were on antihypertensive medications. $75 \%$ of control patients were on antihypertensive medication while the percentage of cases that were on antihypertensive medication was 62.5 . BP was controlled only in $28 \%$ of cases who were on antihypertensive medication. BP was relatively better managed in control group, where it was controlled in $56.67 \%$ of treated patients. Left ventricular hypertrophy, decreased in renal function, microalbuminuria and retinopathy all were significantly higher in patients who were not on treatment and who were on treatment but BP was not controlled( Fig. 1).

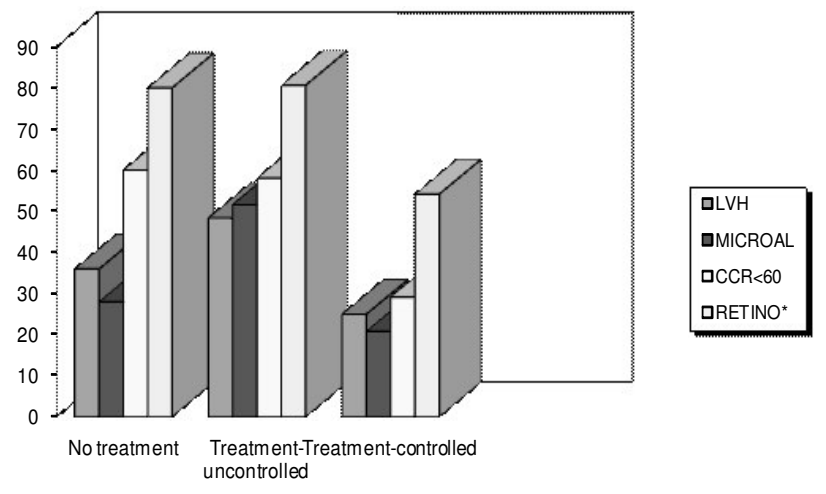

Fig. 1: Prevalence of TOD according to treatment groups 


\section{Discussion}

This study demonstrates that mild reduction in creatinine clearance rate is associated with subclinical end-organ damage, namely, LVH, retinal vascular changes and microalbumin, in a population with primary hypertension and normal serum creatinine levels.

These data are relevant to previous reports, which showed an association between mild renal dysfunction and adverse cardiovascular prognosis in hypertensive patients. In the 'Hypertension Detection and Follow-up Program trial', a linear relation between serum creatinine and cardiovascular mortality was observed over a 5-year follow-up, with a 2 times higher risk of mortality in patients with serum creatinine $>1.7 \mathrm{mg} / \mathrm{dl}$ at baseline. ${ }^{4}$

More recently, in the HOT study a slight increase in serum creatinine was associated with a 3.2 times greater incidence of fatal events, regardless of common cardiovascular risk factors. ${ }^{5}$ Similar findings have also been reported by using creatinine clearance as an estimate of the GFR in patients with isolated systolic hypertension and in those with concomitant cardiovascular risk factors participating in the HOPE study. ${ }^{6,7}$

Among the quantitative markers of target organ damage (TOD), the prognostic significance of echocardiographically confirmed LVH in hypertension is well established. ${ }^{8} \mathrm{LVH}$ is widely considered as "the hemoglobin A1C of blood pressure" since it is an objective measure of both the severity and duration of the elevation in BP. ${ }^{9}$

The prevalence of $\mathrm{LVH}$ was $20 \%$ in patients with $\mathrm{CCR}<60$ and $55 \%$ in patients with CCR $<60(\mathrm{p}=.001)$. This value is slightly lower than that shown by Giovanna Leoncini et al (42\% VS 71\%, $\mathrm{p}=0.0001) .{ }^{10}$ Cesare Cuspidi et al showed $22 \%$ prevalence of $\mathrm{LVH}^{11}{ }^{11}$ The prevalence of LVDD in patients not having LVH was $20 \%$ in patients with CCR $<60$ and $27.5 \%$ in patients with CCR $<60$. Fagard R et al found evidence of asymptomatic LVDD in $33 \%$ of patients who did not have LVH. ${ }^{12}$

There was positive significant correlation between LVH and microalbuminuria $(\mathrm{r}=.677, \mathrm{p}=.000)$, duration of hypertension $(\mathrm{r}=.352, \mathrm{p}=.001)$, smoking $(\mathrm{r}=.452, \mathrm{p}=.000)$ and uncontrolled $\mathrm{BP}(\mathrm{r}=.314, \mathrm{p}=.019)$. There is negative significant correlation between LVH and CCR ( $\mathrm{r}=-0.361, \mathrm{p}=001)$.

Similarly, the prevalence of microalbuminuria in this study is $20 \%$ in control group and $50 \%$ in cases $(p=.005)$. Though these values are higher than that shown by Giovanna Leoncini et al, it is consistent with that shown by Hsegholm A et al and Bigazzi R et al. In their study, the prevalence of micralbuminuria was in the range of $25 \%$ to $40 \%$ in primary hypertensive patients selected from a hypertension clinic. ${ }^{10-}$ 14

Patients with microalbuminuria showed higher prevalence of LVH (78.57\% vs $15.38 \%, \mathrm{p}=.000)$. It was more strongly correlated with LVH than the correlation between CCR and LVH ( $\mathrm{r}=0.677 \mathrm{VS} \mathrm{r}=-0.361)$. Cesare Cuspidi et al showed positive significant correlation between microalbuminuria and left ventricular mass $(\mathrm{r}=0.20, \mathrm{p}<0.03){ }^{11}$

In this study, we found negative significant correlation between microalbuminuria and CCR $(r=-0.314, p=.004)$. This finding is consistent with results observed by De La Sierra et al who showed a serum creatinine $>88 \mathrm{pmol} / \mathrm{L}$ (odds ratio: 3.08; CI 95\%: 1.39-6.84) was independently associated with increased urinary albumin excretion. ${ }^{16}$ Cesare Cuspidi et al showed different grade of retinopathy in $80.5 \%$ of patients. ${ }^{11}$ G. MULÈ et al showed higher prevalence of hypertensive retinopathy in patients with HTN and metabolic syndrome (87.7\% vs. $48.4 \% ; P<0.00001){ }^{15}$

In this study we found $92.5 \%$ retinopathy of different grade in patients with $\mathrm{CCR}<60$ and $52.5 \%$ retinopathy in patients with CCR e" 60 ( $p=.001)$. Its prevalence was also significantly higher in smokers $(\mathrm{p}=.011)$, in patients with microalbuminuria $(\mathrm{p}=.001)$, in patients with uncontrolled BP $(\mathrm{p}=.035)$ and in patients with longer duration of HTN $(\mathrm{P}=.023)$.

\section{Conclusions}

A reduction in creatinne clearance is a marker of sub-clinical organ damage in patients with primary hypertension and normal serum creatinine irrespective of BP load and other traditional risk factors. Thus, due to the ready availability and low cost, this test can be routinely performed not only to assess renal function but also to obtain a more sensitive evaluation of cardiovascular damage in hypertensive patients.

\section{References}

1. Gu D, Reynolds K, Wu X, et al: Prevalence, awareness, treatment, and control of hypertension in China. Hypertension 40:920, 2002.

2. Lloyd-Jones DM, Evans JC, et al: Treatment and control of hypertension in the community: A prospective analysis. Hypertension 40:640, 2002.

3. ESH-ESC Guidelines Committee. 2003 European Society of Hypertension-European Society of Cardiology Guidelines for the management of arterial hypertension. J Hypertens 2003; 21:1011-1053. 
4. Shulman NB et al. Prognostic value of serum creatinine and effect of treatment of hypertension on renal function. Results from the hypertension detection and follow-up program. Hypertension 1989: 13: 180-193.

5. Ruilope LM et al. Renal function and intensive lowering of blood pressure in hypertensive participants of the hypertension optimal treatment (HOT) study. J Am Soc Nephrol 2001; 12: 218-225.

6. Pahor $M$ et al. Diuretic-based treatment and cardiovascular events in patients with mild renal dysfunction enrolled in the systolic hypertension in the elderly program. Arch Intern Med 1998; 158: 13401345 .

7. JF et al. Renal insufficiency as a predictor of CV outcomes and the impact of ramipril: the HOPE randomized trial. Ann Intern Med 2001; 134: 629-636.

8. Muiesan ML et al: Association of change in LV mass with prognosis during long-term antihypertensive treatment. J Hypertens 1995; 13: 1091-5.

9. Cuspidi C, Lonati L et al: Prevalence of target organ damage in treated hypertensive patients: different impact of clinical and ambulatory blood pressure control. J Hypertens 2000; 18: 803-9.

10. Giovanna Leoncini et al. Mild Renal Dysfunction and Subclinical Cardio- vascular Damage in Primary Hypertension. Hypertension 2003;42; 14-18.

11. C Cuspidi et al Evaluation of TOD in arterial hypertension: which role for qualitative funduscopic examination? Ital Heart J Vol 2 September 2001.

12. Fagard R et al: Left ventricular diastolic function predicts outcome in uncomplicated hypertension. Am J Hypertens 2001 Jun; 14(6 Pt 1): 504-8.

13. Hsegholm A et al. Microalbuminuria in 411 untreated individuals with established hypertension, white coat hypertension, and normotension. Hypertension 1994; 24:101-5.

14. Bigazzi R et al. Prevalence of microalbuminuria in a large population of patients with mild to moderate essential HTN. Nephron 1992; 61: 94-7.

15. Christoph D Gatzka et al. Left ventricular mass and microalbuminuria: relation to ambulatory blood pressure. Clin Exp Pharmacol and Physiol, Vol 26 Page 514 - July 1999.

16. De La Sierra et al: Microalbuminuria in essential hypertension: Clinical and biochemical profile. British Journal of Biomedical Science, 2000. 